\title{
Association of gut microbial communities with plasma lipopolysaccharide-binding protein (LBP) in premenopausal women
}

\author{
Jessica S Citronberg ${ }^{1,2} \cdot$ Keith R Curtis ${ }^{2}$ Emily White ${ }^{1,2} \cdot$ Polly A Newcomb ${ }^{1,2} \cdot$ Katherine Newton $^{3}$. \\ Charlotte Atkinson ${ }^{4} \cdot$ Xiaoling Song $^{2} \cdot$ Johanna W Lampe $^{1,2} \cdot$ Meredith AJ Hullar $^{2}$
}

Received: 27 June 2017 / Revised: 8 November 2017 / Accepted: 12 January 2018 / Published online: 12 February 2018

(c) International Society for Microbial Ecology 2018

\begin{abstract}
The mechanisms by which obesity increases cancer risk are unclear, but some lines of evidence suggest that gut microbial communities (GMC) may contribute to chronic inflammation in obese individuals through raised systemic levels of lipopolysaccharides (LPS). We evaluated associations of the GMC in stool with plasma LPS-binding protein (LBP, a measure of LPS) and C-reactive protein (CRP) concentrations in 110 premenopausal women in the United States. Diet was assessed using 3-day food records and GMCs were evaluated using pyrosequencing of the 16S rRNA gene. OTUs were identified at $97 \%$ sequence similarity. Taxonomic classification and functional genes were imputed from 16S rRNA genes, and alpha and beta diversity were assessed using the Shannon index and MRPP, respectively. Multivariable linear regression analysis was used to assess the relation between LBP, specific bacterial genera identified with indicator species analysis, and CRP. Dietary fat intake, particularly saturated fat, and CRP were positively associated with increased LBP. GMC beta diversity, but not alpha diversity, was statistically significantly different between groups using unweighted Unifrac. Several taxa, particularly those in the Clostridia class, were more prevalent in women with low LBP, while Bacteroides were more prevalent in those with high LBP. Genes associated with gram-negative cell wall material synthesis were also associated with LBP and CRP. In contrast, Phascolarctobacterium was associated with lower concentrations of LBP and CRP. We found distinct differences between tertiles of LBP regarding the diversity and composition of the microbiome, as well as differences in functional genes that potentially activate LBP.
\end{abstract}

\section{Introduction}

Current evidence suggests a possible role of gut microbiota in the pathogenesis of obesity and its concomitant diseases, including several cancers [1-3]. Obesity, especially adiposity, is associated with systemic microinflammation (i.e., chronic low-grade inflammation) [1]. Furthermore, adipocytes and macrophages infiltrating visceral adipose tissue in

Jessica S Citronberg

jcitronb@fredhutch.org

1 Department of Epidemiology, University of Washington, Seattle, WA, USA

2 Public Health Sciences Division, Fred Hutchinson Cancer Research Center, Seattle, WA, USA

3 Group Health Research Institute, Seattle, WA, USA

4 NIHR Bristol Biomedical Research Centre (Nutrition Theme) at the University Hospitals Bristol NHS Foundation Trust and the University of Bristol, Bristol, UK obese individuals are a source of circulating proinflammatory cytokines, such as TNF- $\alpha$, IL-1, and IL-6 $[1,4,5]$. More recently, research has suggested that an alteration in gut microbial communities (GMC) escalates systemic microinflammation, at least in obese individuals [6].

Lipopolysaccharide (LPS), a cell wall component of gram-negative bacteria, has been identified as an underlying factor of obesity-driven low-grade inflammation [7]. Lipopolysaccharide-binding protein (LBP), a protein that binds to LPS and transfers LPS monomers to CD14, is driven by circulating concentrations of LPS [8]. Exposure to LPS induces an increase of LBP production in the liver within 15-30 min [9], with a maximum serum level occurring after 24-48 h [10]. The LPS-LBP-CD14-MD2 complex elicits a pro-inflammatory response by Toll-like receptor 4 (TLR4)-mediated NF- $\mathrm{KB}$ activation [11], and, as such, circulating levels of LBP have been found to be associated with systemic inflammation [12]. C-reactive protein (CRP), a biomarker of inflammation, is associated with increased LPS [13]. 
Recent studies have shown that LBP, a marker of LPS exposure, is associated with high-fat diets and obesity [7, 14-17]; however, relatively few studies have examined whether LBP levels vary by GMC distribution and adiposity. Previous reports have shown a strong association between concentrations of circulating LBP, obesityassociated metabolic disturbances, and increased inflammatory signaling [7, 18, 19]. Moreover, LPS-driven inflammation may play a role in the pathogenesis of several adverse outcomes, including inflammatory bowel disease [20-22] and cancer [23, 24]. Nonetheless, the interplay between obesity, GMC, LBP, and inflammation has yet to be elucidated. The goal of the proposed study was to examine whether GMC varies by LBP concentrations, whether there are specific genera associated with varying levels of LBP, and whether these genera are associated with inflammation, as measured by CRP concentrations.

\section{Methods}

\section{Study participants}

Participants were from the Equol, Breast and Bone (EBB) study, which was designed to evaluate the relationship between bacterial metabolic phenotypes, diet, and other exposures, and biomarkers of sex steroid hormone status, has been previously described [25]. Briefly, participants in the EBB study were recruited from the Group Health Cooperative (GHC), a large mixed-model health care system in Washington State. Women were eligible if they were premenopausal, aged 40-45 years, and had received a screening mammogram at GHC prior to recruitment. Women were ineligible to participate if they were allergic to soy beans or soy protein; had been diagnosed with Crohn's disease or ulcerative colitis or had any part of their colon removed; had been diagnosed with breast cancer; were pregnant or planning to become pregnant; had a hysterectomy or any part of their ovaries removed; were perimenopausal (skipped $\geq 1$ periods in the previous 12 months, or had irregular bleeding patterns); were currently using hormone therapy or oral contraceptive, had used them for $\geq 1$ month in the past 12 months, or had used them in the 6 months before their screening mammogram; were currently taking antibiotics or had taken them for $\geq 1$ month in the previous 12 months; or had ever taken tamoxifen or were currently taking raloxifene, bisphosphonates, or oral steroids.

After obtaining informed consent, EBB participants were mailed a health and demographics questionnaire and a physical activity questionnaire, to be completed prior to their clinic visit. At the clinic visit, weight and height were measured as well as body fat distribution (adiposity \%), which was assessed using dual energy X-ray absorptiometry (DXA; Hologic Delphi, Hologic Inc.). Participants also provided a $12 \mathrm{~h}$ fasting blood sample at the clinic visit. All samples were processed within $1 \mathrm{~h}$ of collection, aliquoted into $1.8-\mathrm{ml}$ tubes, and stored at $-70^{\circ} \mathrm{C}$. Date, time of collection, and time since last meal were recorded. Stool sample collection was optional. Study participants were asked to place a sample in RNAlater and samples were stored at $-80^{\circ} \mathrm{C}$ when received by the lab, as previously described [26, 27]. Additionally, all participants were asked to complete a 3-day food record (3DFR) within 2 weeks of the clinic visit.

A total of 1407 women were identified as potential participants from the Group Health Breast Cancer Screening Program. Of these women, $367(26 \%)$ were found to be ineligible, 691 (49\%) refused participation, and $146(10 \%)$ were not able to be interviewed or scheduled for a clinic visit. Of the 203 EBB study participants, 110 completed a health questionnaire, provided stool and blood samples, and had body fat $\%$ measured from a DXA scan.

\section{Biological specimens}

LBP concentrations were measured in plasma using a commercial ELISA kit (Cell Sciences Inc): samples were diluted 1:1000 and the assay was conducted per kit protocol with a standard curve of $5-50 \mathrm{ng} / \mathrm{mL}$. Serum CRP was measured using CRP Ultra Wide Range reagent (Genzyme Diagnostics) on a Roche Cobas Mira chemistry analyzer and read at $570 \mathrm{~nm}$. Samples were run in duplicate, and the mean duplicate intra-assay coefficients of variation $(\mathrm{CV})$ were: $4.7 \%$ for LBP and $4.1 \%$ for CRP. A blinded and pooled plasma sample was included in each batch to track plate-to-plate variation. The inter-batch $\mathrm{CV}$ was $12.5 \%$ for LBP and the assay was conducted on once-thawed samples.

\section{Microbiome bioinformatics analysis}

DNA extraction and 16S rRNA gene sequencing methods used on EBB samples have been previously described [26]. Briefly, DNA was extracted from stool that had been stored in RNAlater. The V1-V3 region of the 16S rRNA gene was amplified and sequenced using 454 pyrosequencing primers $27 \mathrm{f}$ and 519r. Sequences were compiled and processed using QIIME (v.1.8) [28]. Sequences were removed if they were $<200 \mathrm{bp}$ or $>700 \mathrm{bp}$, had homopolymers $>6 \mathrm{bp}$, more than one mismatch to the forward primer, more than one mismatch to the barcode, or more than six ambiguous bases. Sequences were truncated with a quality score sliding window of size $50 \mathrm{bp}$ using a threshold of 25 . Initial operational taxonomic unit (OTU) generation, wherein microorganisms were grouped by DNA sequence similarity of a specific taxonomic marker gene, was done using 
UCLUST in QIIME at 97\% similarity [29]. The OTU table was filtered using the QIIME script filter_otus_from_otu_table.py with --min_count_fraction set to 0.00005 as recommended in Navas-Molina et al. [30]. An additional filtering step set entries in the OTU table to zero if the number of observations was less than 10 per sample, per OTU. Additional OTU entries were filtered out if they were detected as chimeras using QIIME's identify_chimeric_seqs.py script with method blast_fragments. Sequences were aligned to the Silva $16 \mathrm{~S}$ rRNA gene reference alignment using the NAST algorithm [31]. Sequences that did not align to the appropriate $16 \mathrm{~S}$ rRNA gene region were removed. The sequences were classified using MOTHUR's naive Bayesian Classifier trained against the SILVA database (release 111) clustered at the 97\% similarity level [32]. Classified sequences were assigned to phylum and genuslevel phylotypes to characterize the community structure. Rarefaction was performed as uneven sampling depth biases alpha (within-person) and beta (between-person) diversity estimates. Two participants were dropped after OTUs were rarefied to 1578 sequences per sample.

\section{Analysis of GMC functional profiles}

Profiling phylogenetic marker genes, such as the 16S rRNA gene (as outlined above), are needed to understand the distribution of microbial communities, but does not provide direct evidence of a GMC's functional capabilities. We used PICRUSt [33] to predict the metagenomic contribution of genes whose products may influence LBP activation namely, LPS biosynthesis and lipoteichoic acid (LTA) biosynthesis (a marker of gram-positive bacteria) in our study population. PICRUSt has been utilized previously to describe differences in potential function within human samples and positively corresponds to actual metagenomic and metabolic comparisons [33].

\section{Statistical analysis}

Differences in baseline characteristics (age, education, dietary variables, physical activity, body fat, and CRP) were calculated using ANOVA. Diversity of the microbial community within an individual (alpha diversity) was calculated from OTUs (at 3\% divergence) using the Shannon index. Differences in phylum, functional genes, and Shannon index values between tertiles of LBP were calculated using ANOVA. Beta diversity estimates that represent the similarity (or difference) in organismal composition between subjects were based on weighted and unweighted UniFrac distance matrices [34]. Unweighted UniFrac estimates the presence or absence of OTUs between individuals while weighted UniFrac is a quantitative measure based on relative abundance of OTUs between individuals. Given that these metrics may be viewed as complementary approaches that explore different aspects of how communities vary between individuals, both weighted and unweighted Unifrac matrices were used in the analysis.

Multiple response permutation procedure (MRPP) [35] was conducted to test whether the GMC composition differed between tertiles of LBP. MRPP was performed with 1000 permutations on weighted and unweighted UniFrac distance matrices. Differences in the GMC by tertiles of LBP concentration were visualized by NMS ordination plots.

Indicator species analysis (ISA) [36] complemented MRPP by assigning significant indicator values to bacteria taxa (at the genera level) that were indicative of community structure separation between LBP tertiles. To adjust for differences in per-subject sampling frequency, $P$ values were averaged over 1000 bootstrap iterations with even persubject sampling frequency. Bootstrapped $P$ values were adjusted for FDR using the Benjamini and Hochberg $(\mathrm{B}-\mathrm{H})$ method [37] based on the total number of taxa after excluding those that represented $<1 \%$ average relative abundance.

The Kruskal Wallis (K-W) tests were used to assess whether phyla and abundance of functional genes (lipopolysaccharide biosynthesis and LTA synthesis) differed by tertile of LBP.

To test the association between LBP and inflammation, a binary CRP value was created to represent CRP concentrations $>1 \mathrm{mg} / \mathrm{L}$ vs. $\leq 1 \mathrm{mg} / \mathrm{L}$. The cutoff value of $1 \mathrm{mg} /$ L was based on clinical guidelines [38] and previous studies [39]. Unconditional logistic regression was used to compute odds ratios (ORs) and 95\% confidence intervals (CIs) for the relationship between LBP, the exposure variable, and CRP, the outcome variable. Additional adjustment for a priori confounders (smoking status and age) was performed in the multivariable model.

Additionally, regression models adjusting for smoking status and age were run to test the association between taxa identified in the ISA analysis (categorized into tertiles) and circulating CRP (dichotomized as $(>1 \mathrm{mg} / \mathrm{L}$ and $\leq 1 \mathrm{mg} / \mathrm{L}$ ). Rare taxa, those that were present in less than half the population, were not included in the analysis.

All reported $P$ values are two-sided, and a $P$ value $<0.05$ was considered statistically significant. All analyses were carried out using STATA 14 (StataCorp, College Station, TX), QIIME [40], R version 3.3.1 (http://www.r-project. org), vegan [41], ggplot2 [42], and labdsv [43].

\section{Results}

Characteristics of the study population and macronutrient intakes by tertiles of LBP concentrations are shown in 
Table 1 Selected baseline characteristics of EBB participants by LBP tertile

\begin{tabular}{|c|c|c|c|c|}
\hline & $\begin{array}{l}\text { Tertile } 1 \\
(0-14.9 \mu \mathrm{g} / \\
\mathrm{mL}) \\
n=36\end{array}$ & $\begin{array}{l}\text { Tertile } 2 \\
(15.0-22.1 \mu \mathrm{g} / \mathrm{mL}) \\
n=37\end{array}$ & $\begin{array}{l}\text { Tertile } 3 \\
(22.4-94.7 \mu \mathrm{g} / \mathrm{mL})\end{array}$ & $\begin{array}{l}P \text { for difference } \\
\text { between groups* }\end{array}$ \\
\hline & & & & \\
\hline Age, years & $42.34(1.3)$ & $42.54(1.4)$ & $42.33(1.4)$ & 0.82 \\
\hline Ethnicity & & & & 0.17 \\
\hline White & $34(97.1 \%)$ & $30(83.3 \%)$ & $34(94.4 \%)$ & \\
\hline Asian & $0(0.0 \%)$ & $3(8.3 \%)$ & $1(2.8 \%)$ & \\
\hline Other & $1(2.9 \%)$ & $3(8.3 \%)$ & $1(2.8 \%)$ & \\
\hline Education & & & & 0.54 \\
\hline$\leq 12$ years & $0(0.0 \%)$ & $3(9.1 \%)$ & $2(6.3 \%)$ & \\
\hline $13-15$ years & $9(27.3 \%)$ & $6(18.2 \%)$ & $10(31.3 \%)$ & \\
\hline 16 years & $10(30.3 \%)$ & $10(30.3 \%)$ & $10(31.3 \%)$ & \\
\hline $17+$ years & $14(42.4 \%)$ & $14(42.4 \%)$ & $10(31.3 \%)$ & \\
\hline $\begin{array}{l}\text { Energy intake (kcal/ } \\
\text { day) }\end{array}$ & $1899(343)$ & $1,871(446)$ & $2,020(437)$ & 0.27 \\
\hline Protein (g/day) & $78.3(19.2)$ & $76.4(17.7)$ & $79.1(18.8)$ & 0.82 \\
\hline Carbohydrate (g/day) & $231.5(62.6)$ & $228.1(64.9)$ & $239.9(61.7)$ & 0.71 \\
\hline Total fat (g/day) & $71.9(18.0)$ & $71.7(22.3)$ & $81.2(24.9)$ & 0.11 \\
\hline$\%$ Energy from fat & $34.4(8.3)$ & $34.2(6.1)$ & $35.5(6.6)$ & 0.73 \\
\hline Saturated fat (g/day) & $24.4(7.9)$ & $25.4(3.8)$ & $29.0(10.8)$ & 0.10 \\
\hline Dietary fiber (g/day) & $21.1(7.9)$ & $19.3(7.3)$ & $21.1(8.2)$ & 0.54 \\
\hline Soluble fiber (g/day) & $5.3(2.0)$ & $5.0(2.1)$ & $5.6(2.4)$ & 0.72 \\
\hline Insoluble fiber (g/day) & $15.6(6.1)$ & $14.1(5.5)$ & $15.3(6.4)$ & 0.66 \\
\hline Alcohol (g/day) & $8.3(12.3)$ & $7.2(10.5)$ & $8.8(13.4)$ & 0.49 \\
\hline History of smoking & & & & 0.88 \\
\hline Yes & $12(34.3 \%)$ & $11(30.6 \%)$ & $13(36.1 \%)$ & \\
\hline No & $23(65.7 \%)$ & $25(69.4 \%)$ & $23(63.9 \%)$ & \\
\hline $\begin{array}{l}\text { Physical activity } \\
\text { (METS/year) }\end{array}$ & $79.52(44.9)$ & $105.06(67.01)$ & $95.92(73.87)$ & 0.63 \\
\hline $\begin{array}{l}\text { Percent body fat } \\
\text { (tertiles) }\end{array}$ & & & & 0.18 \\
\hline $0(<29.4 \%)$ & $15(45.5 \%)$ & $12(36.4 \%)$ & $7(21.9 \%)$ & \\
\hline $1(29.5-37.3 \%)$ & $11(33.3 \%)$ & $8(24.2 \%)$ & $12(37.5 \%)$ & \\
\hline $2(>37.3 \%)$ & $7(21.2 \%)$ & $13(39.4 \%)$ & $13(40.6 \%)$ & \\
\hline CRP (mg/L) & $0.95(1.4)$ & $1.4(2.26)$ & $2.9(3.8)$ & 0.002 \\
\hline
\end{tabular}

$* P$ value calculated using ANOVA

Table 1. No substantial differences in ethnicity, education, smoking history, age, and dietary fiber intake were observed across tertiles. CRP was statistically significantly different between tertiles of LBP $(P=0.002)$, with higher tertiles corresponding to higher CRP values. Compared to those in the lowest tertile, those in the highest tertile of LBP had a higher total fat intake, saturated fat intake, and \% body fat, although these differences were not statistically significant.

Bacteria were distributed across phyla: Actinobacteria $(0.2 \%)$, Bacteroidetes $(30.4 \%)$, Cyanobacteria $(0.03 \%)$, Firmicutes $(67.7 \%)$, Lentispharae $(0.06 \%)$, Proteobacteria $(1.2 \%)$, and Tenericutes $(0.4 \%)$, and Verrucomicrobia
$(0.03 \%)$ (Table 2). Firmicutes decreased while Bacteroidetes increased with LBP concentration although these differences were not statistically significant. Additionally, the relative abundance of Actinobacteria was statistically significantly different between the three LBP groups $(P=$ 0.03). Moreover, imputed genes for LPS biosynthesis were statistically significantly different by tertiles of LBP (tertile 1: 254.8, tertile 2: 287.6, tertile 3: 339; $P<0.0001)$. However, genes in the LTA biosynthesis pathway were not statistically significantly different between groups.

The bacterial alpha diversity measured by the Shannon index was not significantly different between tertiles of 
Table 2 GMC distribution of EBB participants by LBP tertile

\begin{tabular}{llll} 
Tertile 1 & Tertile 2 & Tertile 3 & $\begin{array}{l}P \text { for } \\
\text { difference } \\
\text { between } \\
\text { groups }^{\mathrm{a}}\end{array}$ \\
$n=36$ & $n=37$ & $n=37$ & \\
\hline
\end{tabular}

\begin{tabular}{lllll}
\hline Phylum (\%) & & & & \\
Actinobacteria & 0.1 & 0.22 & 0.27 & 0.03 \\
Bacteroidetes & 34.6 & 27.0 & 29.6 & 0.14 \\
Cyanobacteria & 0.0 & 0.07 & 0.005 & 0.36 \\
Firmicutes & 63.8 & 70.9 & 68.3 & 0.16 \\
Lentispharae & 0.05 & 0.08 & 0.04 & 0.98 \\
Proteobacteria & 1.2 & 1.2 & 1.2 & 0.51 \\
Tenericutes & 0.2 & 0.6 & 0.5 & 0.25 \\
$\quad$ Verrucomicrobia & 0.02 & 0.03 & 0.03 & 0.77 \\
$\quad$ Other & 0.007 & 0.0 & 0.004 & 0.36 \\
Functional genes & & & & \\
& 254.8 & 287.6 & 339.0 & $<0.0001$ \\
Lipopolysaccharide & & & & \\
synthesis & & & & \\
$\quad$ LTA synthesis & 210 & 290.7 & 305.2 & 0.08 \\
Alpha diversity & & & & \\
Shannon index & 4.58 & 4.46 & 4.66 & $0.71^{*}$ \\
\hline
\end{tabular}

${ }^{\mathrm{a}}$ Based on $\mathrm{K}-\mathrm{W}$ test

$* P$ value calculated using ANOVA

plasma LBP (tertile 1: 4.58; tertile 2: 4.46; tertile 3: 4.66, ANOVA, $F=0.3356, n=110, P=0.71$; Table 2). Beta diversity was significantly different between tertiles of LBP using unweighted Unifrac (MRPP; $A=0.005, P=0.027$, 1000 permutations), but no difference between tertiles of LBP was observed using weighted Unifrac (MRPP; $A=$ $0.0002919, \quad P=0.20 ; 1000$ permutations; figure not included).

Using indicator species analysis (ISA), we found that 16 bacterial OTUs were associated with LBP. Of these, 14 phylotypes were "indicators" of low levels of LBP concentrations, and two were indicators for the highest tertile of LBP (Table 3). Uncultured Christensenellaceae and unclassified Ruminococcaceae showed the highest indicator values for low LBP, while Bacteroides was the strongest indicator for high LBP.

In the unadjusted model, LBP was statistically significantly associated with $\mathrm{CRP}>1 \mathrm{mg} / \mathrm{L}\left(P_{\text {trend }}=0.02\right)$ and the OR among those in the third tertile for LBP (22.2-94.7 $\mu \mathrm{g} / \mathrm{mL}$ ) was statistically significantly higher than that in the lowest tertile $(\mathrm{OR}=2.98 ; 95 \% \mathrm{CI}=1.14-7.80)$. However, in the adjusted model, there was no observed association among those in the highest (third) LBP quartile (OR = 2.09; $95 \% \mathrm{CI}=0.76-5.80)$ and the test for trend was not statistically significant $\left(P_{\text {trend }}=0.15\right)$. In both the crude and the adjusted model, the association between Bacteroides and
CRP $>1 \mathrm{mg} / \mathrm{L}$ increased across tertiles of Bacteroides, with a non-statistically significant trend observed across the three groups $\left(P_{\text {trend }}=0.08\right)$.

Compared to no Phascolarctobacterium present (first tertile), high levels of Phascolarctobacterium (third tertile) were statistically significantly associated with CRP $\leq 1 \mathrm{mg} /$ $\mathrm{L}(\mathrm{OR}=0.30,95 \% \mathrm{CI}: 0.11-0.81)$; with a monotonic trend observed across the three groups $\left(P_{\text {trend }}=0.02\right)$ in the adjusted model (Table 4$)$. For the remaining genera, overall bacteria levels were not statistically significantly associated with CRP $>1 \mathrm{mg} / \mathrm{L}$ in either the crude or the multivariable adjusted models.

\section{Discussion}

Microbial mechanisms have been linked to inflammation that may be associated with increased risk for cancer and other chronic diseases. One such pathway is through dietary-related shifts in the gut microbiome linked to activation of the innate immune response. In this crosssectional study, we found distinct differences in the diversity, and composition of the stool microbiome and some potential functional pathways for synthesis of cell wall material in gram-negative and gram-positive bacteria between tertiles of LBP. Furthermore, study results showed that differences in bacterial genera that potentially activate LBP were associated with increased inflammation.

Diets, particularly high-fat diets, may contribute to the microbiome-inflammation relationship [44, 45]. In this study of premenopausal women, we found a nonstatistically significant positive association between increasing tertiles of circulating LBP and dietary fat intake. Mechanisms associated with a higher systemic LPS load and increased LBP response include (1) increased translocation of LPS via chylomicron uptake and/or (2) increased intestinal permeability associated with diet [6, 46, 47]. A cross-sectional study of healthy men aged 45-64 years, observed that fat intake was significantly associated with increased endotoxin load [48] while other studies have found that high fat/high carbohydrate foods increase LPS load, thus suggesting that diet may influence circulating levels of LPS concentrations [7, 49, 50].

Changes in diet leading to changes in inflammation have been associated with shifts in microbial community structure [51, 52]. Our results extend these findings by showing that the overall composition and functional genes associated with gram-negative cell wall synthesis were associated with higher levels of LBP. We found that the overall gut microbiome composition in individuals with higher circulating concentrations of LBP was different from those with lower levels using unweighted Unifrac, but these findings 
Table 3 Indicator species analysis - genus level

\begin{tabular}{llll}
\hline & LBP tertile & Indicator value & Corrected $P$ value* \\
\hline Firmicutes._Clostridia._Clostridiales._Christensenellaceae._uncultured & 1 & 0.5824 & 0.008 \\
Firmicutes._Clostridia__Clostridiales._Ruminococcaceae.Other & 1 & 0.5619 & 0.008 \\
Firmicutes._Clostridia._Clostridiales._Ruminococcaceae._uncultured & 1 & 0.5067 & 0.008 \\
Firmicutes._Clostridia._Clostridiales._Ruminococcaceae._Subdoligranulum & 1 & 0.4519 & 0.04 \\
Firmicutes._Clostridia._Clostridiales._Ruminococcaceae._Incertae_Sedis._human gut & 1 & 0.4494 & 0.04 \\
metagenome & & 1 & 0.4322 \\
Firmicutes._Clostridia._Clostridiales._Veillonellaceae._Phascolarctobacterium & 1 & 0.4032 & 0.008 \\
Firmicutes._Clostridia._Clostridiales._Ruminococcaceae._Ruminococcus & 1 & 0.3925 & 0.04 \\
Tenericutes._Mollicutes._RF9._uncultured_bacterium.Other & 1 & 0.3711 & 0.01 \\
Bacteroidetes._Bacteroidia._Bacteroidales.__Porphyromonadaceae._Barnesiella & 1 & 0.3529 & 0.008 \\
Firmicutes._Clostridia._Clostridiales._uncultured._uncultured_bacterium & 1 & 0.3510 & 0.008 \\
Firmicutes._Clostridia._Clostridiales._Ruminococcaceae._Anaerotruncus & 1 & 0.3329 & 0.04 \\
Firmicutes._Clostridia._Clostridiales._Ruminococcaceae._Oscillibacter & 1 & 0.3056 & 0.01 \\
Tenericutes._Mollicutes._RF9.Other.Other & 1 & 0.2102 & 0.04 \\
Firmicutes._Clostridia._Clostridiales._uncultured._uncultured.Other & 3 & 0.4713 & 0.02 \\
Bacteroidetes._Bacteroidia._Bacteroidales._Bacteroidaceae._Bacteroides & 3 & 0.1351 & 0.04 \\
Bacteria._Firmicutes._Clostridia._Clostridiales._Lachnospiraceae._Moryella & &
\end{tabular}

*Benjamini-Hochberg corrected $P$ value

were not observed using weighted Unifrac. The discrepancy in results suggest that there may be differences in rarer taxa between tertiles of LBP, as unweighted Unifrac is sensitive to rarer taxa while weighted Unifrac is less influenced by these taxa [53].

Further analysis revealed that several taxa, particularly those found in the gram-positive Clostridia class, may be more prevalent with low levels of LBP while Bacteroides may be more prevalent with high levels of LBP. In the lowest tertile, the highest indicator value belonged to the gram-positive Christensenellaceae genus, which has been found to be more abundant in lean individuals [54]. Animal models have also shown that transplantation of Christensenellaceae into germ-free mice protected against weight gain [55]. Christensenellaceae, one of the most heritable members of the microbiome, has also been inversely associated with obesity in other animal and human studies [56, 57]. Thus, findings suggest that the association between LBP and obesity, may, in part, be driven by the genera (or lack thereof) associated with higher LBP concentrations. Alternatively, the highest indicator value in the highest tertile of LBP was Bacteroides, a gram-negative genus which has been associated with both obesity and inflammatory bowel disease in previous studies [58, 59]. Taken together, these findings point to an association between increased levels of bacteria associated with obesity and inflammation, and increased levels of circulating LBP, supporting the hypothesis that changes in dietary patterns may lead to shifts in the gut microbiome, subsequently leading to obesity and inflammation.
In addition to differences in genera present by LBP, we also observed functional differences in GMC that may be associated with LBP concentrations. LPS biosynthesis, based on pathway genes identified in KEGG, was statistically significantly different by tertiles of LBP. These findings support the hypothesis that the GMC associated with chronic low-grade inflammation may, in part, be driven by gene differences, which can be detected as differences circulating LBP. Alternatively, we did not observe any differences in LTA synthesis genes across LBP tertiles. While previous studies have shown that activation of cellular responses by LTA, a cell wall component of gram-positive bacteria, is enhanced by LBP $[60,61]$, no previous studies have examined the association between LTA genes and circulating LBP levels; current study results suggest that the functional potential of genes-related LTA synthesis is not associated with those responsible for LPS synthesis.

The LPS-LBP-CD14-MD2 complex elicits a proinflammatory response by TLR4-mediated NF- $\kappa \mathrm{B}$ activation [11], and, as such, circulating levels of LBP have been found to be associated with systemic inflammation [12]. CRP, a biomarker of inflammation, has also been shown to be associated with increased LPS [13]. Consistent with previous studies that have observed an association between LBP and inflammation [14, 62], our study showed that LBP concentrations were associated with inflammation (as measured by CRP), but only adding covariates to the model. Failure to observe an association in the multivariable model may be due to mediation effects of LBP on the association 
Table 4 Estimated odds ratios (OR) of CRP associated with the microbiome

\begin{tabular}{|c|c|c|c|c|}
\hline $\begin{array}{l}\text { Microbiome } \\
\text { measurement } \\
\text { (tertiles) }\end{array}$ & Range & $N$ & $\begin{array}{l}\text { OR } \\
\text { No adjustment }\end{array}$ & $\begin{array}{l}\text { OR } \\
\text { Multivariate }_{\text {adjusted }^{\mathrm{a}}}\end{array}$ \\
\hline \multicolumn{5}{|l|}{ LBP tertiles } \\
\hline 1 & $6.8-14.9 \mu \mathrm{g} / \mathrm{mL}$ & 36 & Ref. & Ref. \\
\hline 2 & $15.0-22.1 \mu \mathrm{g} / \mathrm{mL}$ & 37 & $0.96(0.35-2.61)$ & $0.95(0.34-2.67)$ \\
\hline \multirow[t]{2}{*}{3} & $22.2-94.7 \mu \mathrm{g} / \mathrm{mL}$ & 37 & $2.98(1.14-7.80)$ & $2.09(0.76-5.80)$ \\
\hline & & & $P_{\text {trend }} 0.02$ & $P_{\text {trend }} 0.15$ \\
\hline \multicolumn{5}{|c|}{ Bacteroidaceae_Bacteroides } \\
\hline 1 & $0.009-0.117$ & 37 & Ref. & Ref. \\
\hline 2 & $0.118-0.216$ & 37 & $2.05(0.78-5.45)$ & $2.21(0.78-6.20)$ \\
\hline \multirow[t]{2}{*}{3} & $0.234-0.562$ & 36 & $2.41(0.91-6.41)$ & $2.52(0.90-7.10)$ \\
\hline & & & $P_{\text {trend }} 0.08$ & $P_{\text {trend }} 0.08$ \\
\hline \multicolumn{5}{|c|}{ Clostridiales_Christensenellaceae_uncultured } \\
\hline 1 & $0-0.004$ & 37 & Ref. & Ref. \\
\hline 2 & $0.0041-0.049$ & 37 & $0.80(0.32-2.02)$ & $0.84(0.32-2.20)$ \\
\hline \multirow[t]{2}{*}{3} & $0.52-0.25$ & 36 & $0.74(0.29-1.90)$ & $0.96(0.35-2.61)$ \\
\hline & & & $P_{\text {trend }} 0.53$ & $P_{\text {trend }} 0.92$ \\
\hline \multicolumn{5}{|c|}{ Ruminococcacae _Ruminococcus } \\
\hline 1 & $0-0.002$ & 37 & Ref. & Ref. \\
\hline 2 & $0.0021-0.0125$ & 37 & $0.63(0.24-1.62)$ & $0.70(0.26-1.89)$ \\
\hline \multirow[t]{2}{*}{3} & $0.0125-0.063$ & 36 & $0.94(0.37-2.37)$ & $1.23(0.45-3.31)$ \\
\hline & & & $P_{\text {trend }} 0.88$ & $P_{\text {trend }} 0.70$ \\
\hline \multicolumn{5}{|c|}{ Ruminococcacae.Other } \\
\hline 1 & $0.002-0.145$ & 37 & Ref. & Ref. \\
\hline 2 & $0.149-0.038$ & 37 & $1.31(0.52-3.34)$ & $1.49(0.55-4.04)$ \\
\hline \multirow[t]{2}{*}{3} & $0.039-0.147$ & 36 & $0.89(0.35-2.29)$ & $0.84(0.31-2.29)$ \\
\hline & & & $P_{\text {trend }} 0.81$ & $P_{\text {trend }} 0.76$ \\
\hline \multicolumn{5}{|c|}{ Ruminococcaceae_Incertae Sedis_human gut metagenome } \\
\hline 1 & $0-0.007$ & 46 & Ref. & Ref. \\
\hline 2 & $0.0009-0.008$ & 28 & $2.63(0.99-6.97)$ & $2.94(1.02-8.42)$ \\
\hline \multirow[t]{2}{*}{3} & $0.0081-0.14$ & 36 & $1.45(0.58-3.64)$ & $1.50(0.57-3.89)$ \\
\hline & & & $P_{\text {trend }} 0.38$ & $P_{\text {trend }} 0.37$ \\
\hline \multicolumn{5}{|c|}{ Ruminococcaceae_Oscillibacter } \\
\hline 1 & 0 & 57 & Ref. & Ref. \\
\hline 2 & $0.0008-0.004$ & 17 & $1.66(0.56-4.94)$ & $1.59(0.50-5.01)$ \\
\hline \multirow[t]{2}{*}{3} & $0.0041-0.41$ & 36 & $0.65(0.27-1.58)$ & $0.54(0.21-1.38)$ \\
\hline & & & $P_{\text {trend }} 0.41$ & $P_{\text {trend }} 0.24$ \\
\hline \multicolumn{5}{|c|}{ Ruminococcaceae_Subdoligranulum } \\
\hline 1 & $0-0.0095$ & 37 & Ref. & Ref. \\
\hline 2 & $0.0096-0.021$ & 37 & $1.26(0.49-3.30)$ & $1.31(0.47-3.60)$ \\
\hline \multirow[t]{2}{*}{3} & $0.022-0.14$ & 36 & $1.86(0.72-4.82)$ & $1.92(0.71-5.21)$ \\
\hline & & & $P_{\text {trend }} 0.20$ & $P_{\text {trend }} 0.20$ \\
\hline \multicolumn{5}{|c|}{ Ruminococcaceae_Uncultured } \\
\hline 1 & $0-0.008$ & 37 & Ref. & Ref. \\
\hline 2 & $0.005-0.025$ & 37 & $1.12(0.44-2.82)$ & $1.25(0.47-3.35)$ \\
\hline \multirow[t]{2}{*}{3} & $0.027-0.13$ & 36 & $0.73(0.28-1.90)$ & $0.84(0.31-2.31)$ \\
\hline & & & $P_{\text {trend }} 0.53$ & $P_{\text {trend }} 0.75$ \\
\hline
\end{tabular}


Table 4 (continued)

\begin{tabular}{|c|c|c|c|c|}
\hline $\begin{array}{l}\text { Microbiome } \\
\text { measurement } \\
\text { (tertiles) }\end{array}$ & Range & $N$ & $\begin{array}{l}\text { OR } \\
\text { No adjustment }\end{array}$ & $\begin{array}{l}\text { OR } \\
\text { Multivariate }_{\text {adjusted }^{\mathrm{a}}}\end{array}$ \\
\hline \multicolumn{5}{|c|}{ Veillonellaceae_Phascolarctobacterium } \\
\hline 1 & 0 & 49 & Ref. & Ref. \\
\hline 2 & $0.001-0.006$ & 25 & $0.69(0.26-1.84)$ & $0.69(0.24-1.94)$ \\
\hline \multirow[t]{2}{*}{3} & $0.0061-0.026$ & 36 & $0.35(0.14-0.89)$ & $0.30(0.11-0.81)$ \\
\hline & & & $P_{\text {trend }} 0.03$ & $P_{\text {trend }} 0.02$ \\
\hline \multicolumn{5}{|c|}{ Porphyromonadaceae_Barnesiella } \\
\hline 1 & 0 & 50 & Ref. & Ref. \\
\hline 2 & $0.001-0.0046$ & 24 & $0.98(0.37-2.64)$ & $0.97(0.34-2.8)$ \\
\hline \multirow[t]{2}{*}{3} & $0.0047-0.04$ & 36 & $0.69(0.28-1.68)$ & $0.54(0.21-1.40)$ \\
\hline & & & $P_{\text {trend }} 0.43$ & $P_{\text {trend }} 0.22$ \\
\hline
\end{tabular}

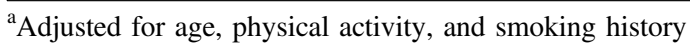

between the covariates and CRP, confounding, or the small sample size.

Phascolarctobacterium, a gram-positive bacterium in the Negativicutes class, was associated with both low LBP concentrations and with low CRP concentrations in our study. Additionally, we found that LBP was associated with a higher dietary fat intake, but not with dietary fiber or carbohydrate intakes. Interestingly, Phascolarctobacterium ferments carbohydrates to the short-chain fatty acid propionate [63], a bacterial metabolite that has anti-lipogenic, cholesterol-lowering, anti-inflammatory, and anticarcinogenic properties [64, 65]. Propionate has been associated with increased satiety, which may be associated with reduced inflammation [66]. These findings complement prior studies, which have shown that Phascolarctobacterium is associated with cruciferous vegetable intake [67] and is significantly reduced in individuals with inflammatory bowel disease [68, 69]. Thus, findings from the current study suggest that LBP is associated with CRP, perhaps through a decrease in gram-positive bacteria that may be associated with reduced inflammation.

Strengths of the study include the use of a well-described population of premenopausal women and stringent exclusion criteria, which removed factors that could potentially influence GMC (e.g., antibiotic and other medication use). Additionally, careful assessment of diet using a 3DFR, rather than a FFQ, reduced the potential for measurement error due to poor recall.

Limitations of our study, beyond the sample size, are the cross-sectional design, and lack of metagenomics data to assess functional genes. The study design cannot measure temporal relationships, and as such, prospective studies are needed to assess whether changes in GMC lead to changes in inflammation. The annotations used in KEGG suggest the functional potential of the community, but the presence of these genes/functions does not mean that they are biologically active (i.e., they may not be transcribed). However, in the current study, an increase in LPS biosynthesis was observed in conjunction with an increase in the gramnegative genera Bacteroides, suggesting that the annotations using KEGG captured the functional gene presence. Forward primer selection may also have influenced results, as previous study has shown that Actinobacteria and Bifidobacterium abundances were underestimated when using the 27f primer [70].

In addition, timing between diet and stool collection may have influenced results as 3DFR were collected within 2 weeks of stool sample collection. While ideally diet and stool samples would be collected at the same time, as changes in dietary patterns can change microbial communities in less than a week [71], a study by $\mathrm{Wu}$ et al. found that short-term dietary changes were modest and did not impact enterotype partitioning established through longterm dietary patterns [72]. Furthermore, while the study presents novel findings based on ISA, independent replication is required as these associations were not established a priori. Results may have been due to chance, given that many taxa were examined, although $p$ values were corrected for multiple testing. Similarly, findings related to ISA genera and CRP may have been due to chance, given the exploratory nature of the analysis and subsequent number of regression models run. Furthermore, given that the study was conducted among premenopausal women, results may not be generalizable to the overall population.

The current study found that the GMC differs in relation to plasma LBP concentrations and the association between LBP and CRP may be due to the presence of certain LPSproducing bacteria that increase LBP concentrations. Our findings point to an association between increased levels of bacteria associated with obesity and inflammation, and 
increased levels of circulating LBP. To our knowledge, this is the first study to examine LBP in relation to CRP and GMC, validation of our findings in larger, prospective studies is needed to establish temporal relationships and whether dietary patterns lead to shifts in the gut microbiome, subsequently leading to obesity and inflammation.

Acknowledgements This work was supported by NIH grants R01 CA97366, U54 CA116847, R03 CA115209, K05 CA154337, R25 CA094880, and Kellogg Corporate Citizens Fund and Fred Hutchinson Cancer Research Center.

\section{Compliance with ethical standards}

Conflict of interest The authors declare that they have no conflict of interest.

\section{References}

1. Hotamisligil GS. Inflammation and metabolic disorders. Nature. 2006;444:860-7.

2. Davis CD, Milner JA. Gastrointestinal microflora, food components and colon cancer prevention. J Nutr Biochem. 2009;20:743-52.

3. Gao Z, Guo B, Gao R, Zhu Q, Qin H. Microbiota disbiosis is associated with colorectal cancer. Front Microbiol. 2015;6:20.

4. Huang C-J, Acevedo EO, Mari DC, Randazzo C, Shibata Y. Glucocorticoid inhibition of leptin- and lipopolysaccharideinduced interleukin- 6 production in obesity. Brain Behav Immun. 2014;35:163-8.

5. Siebler J, Galle PR, Weber MM. The gut-liver-axis: endotoxemia, inflammation, insulin resistance and NASH. J Hepatol. 2008;48:1032-4.

6. Creely SJ, McTernan PG, Kusminski CM, Fisher fM, Da Silva NF, Khanolkar M, et al. Lipopolysaccharide activates an innate immune system response in human adipose tissue in obesity and type 2 diabetes. Am J Physiol Endocrinol Metab. 2007;292: E740-7.

7. Cani PD, Amar J, Iglesias MA, Poggi M, Knauf C, Bastelica D, et al. Metabolic endotoxemia initiates obesity and insulin resistance. Diabetes. 2007;56:1761-72.

8. Schumann RR. Old and new findings on lipopolysaccharidebinding protein: a soluble pattern-recognition molecule. Biochem Soc Trans. 2011;39:989-93.

9. Ramadori G, Meyer zum Buschenfelde KH, Tobias PS, Mathison JC, Ulevitch RJ. Biosynthesis of lipopolysaccharidebinding protein in rabbit hepatocytes. Pathobiology. 1990;58:89-94.

10. Schumann RR, Kirschning CJ, Unbehaun A, Aberle HP, Knope HP, Lamping N, et al. The lipopolysaccharide-binding protein is a secretory class 1 acute-phase protein whose gene is transcriptionally activated by APRF/STAT/3 and other cytokine-inducible nuclear proteins. Mol Cell Biol. 1996;16:3490-503.

11. Schröder NW, Opitz B, Lamping N, Michelsen KS, Zähringer U, Göbel UB, et al. Involvement of lipopolysaccharide binding protein, CD14, and Toll-like receptors in the initiation of innate immune responses by Treponema glycolipids. J Immunol. 2000;165:2683-93.

12. Andreasen AS, Krabbe KS, Krogh-Madsen R, Taudorf S, Pedersen BK, Møller K. Human endotoxemia as a model of systemic inflammation. Curr Med Chem. 2008;15:1697-705.
13. Stehle JR, Leng X, Kitzman DW, Nicklas BJ, Kritchevsky SB, High KP. Lipopolysaccharide-binding protein, a surrogate marker of microbial translocation, is associated with physical function in healthy older adults. J Gerontol A Biol Sci Med Sci. 2012;67:1212-8.

14. Moreno-Navarrete JM, Ortega F, Serino M, Luche E, Waget A, Pardo $\mathrm{G}$, et al. Circulating lipopolysaccharide-binding protein (LBP) as a marker of obesity-related insulin resistance. Int J Obes. 2012;36:1442-9.

15. Gonzalez-Quintela A, Alonso M, Campos J, Vizcaino L, Loidi L, Gude F. Determinants of serum concentrations of lipopolysaccharide-binding protein (LBP) in the adult population: the role of obesity. PLoS ONE. 2013;8:e54600.

16. Sun L, Yu Z, Ye X, Zou S, Li H, Yu D, et al. A marker of endotoxemia is associated with obesity and related metabolic disorders in apparently healthy Chinese. Diabetes Care. 2010;33:1925-32.

17. Ruiz AG, Casafont F, Crespo J, Cayón A, Mayorga M, Estebanez A, et al. Lipopolysaccharide-binding protein plasma levels and liver TNF-alpha gene expression in obese patients: evidence for the potential role of endotoxin in the pathogenesis of nonalcoholic steatohepatitis. Obes Surg. 2007;17:1374-80.

18. Suganami T, Mieda T, Itoh M, Shimoda Y, Kamei Y, Ogawa Y. Attenuation of obesity-induced adipose tissue inflammation in $\mathrm{C} 3 \mathrm{H} / \mathrm{HeJ}$ mice carrying a Toll-like receptor 4 mutation. Biochem Biophys Res Commun. 2007;354:45-9.

19. Blomkalns AL, Stoll LL, Shaheen W, Romig-Martin SA, Dickson EW, Weintraub NL, et al. Low level bacterial endotoxin activates two distinct signaling pathways in human peripheral blood mononuclear cells. J Inflamm. 2011;8:4.

20. Wellmann W, Fink PC, Benner F, Schmidt FW. Endotoxaemia in active Crohn's disease. Treatment with whole gut irrigation and 5aminosalicylic acid. Gut. 1986;27:814-20.

21. Pastor Rojo O, López San Román A, Albéniz Arbizu E, de la Hera Martínez A, Ripoll Sevillano E, Albillos Martínez A. Serum lipopolysaccharide-binding protein in endotoxemic patients with inflammatory bowel disease. Inflamm Bowel Dis. 2007;13:269-77.

22. Gardiner KR, Halliday MI, Barclay GR, Milne L, Brown D, Stephens S, et al. Significance of systemic endotoxaemia in inflammatory bowel disease. Gut. 1995;36:897-901.

23. Fukata M, Chen A, Vamadevan AS, Cohen J, Breglio K, Krishnareddy $\mathrm{S}$, et al. Toll-like receptor-4 promotes the development of colitis-associated colorectal tumors. Gastroenterology. 2007;133:1869-1869.e14.

24. Grivennikov SI, Wang K, Mucida D, Stewart CA, Schnabl B, Jauch D, et al. Adenoma-linked barrier defects and microbial products drive IL-23/IL-17-mediated tumour growth. Nature. 2012;491:254-8.

25. Atkinson C, Newton KM, Bowles EJA, Yong M, Lampe JW. Demographic, anthropometric, and lifestyle factors and dietary intakes in relation to daidzein-metabolizing phenotypes among premenopausal women in the United States. Am J Clin Nutr. 2008;87:679-87.

26. Li F, Hullar MAJ, Schwarz Y, Lampe JW. Human gut bacterial communities are altered by addition of cruciferous vegetables to a controlled fruit- and vegetable-free diet. J Nutr Am Soc Nutr. 2009;139:1685-91.

27. Hullar MAJ, Lancaster SM, Li F, Tseng E, Beer K, Atkinson C, et al. Enterolignan-producing phenotypes are associated with increased gut microbial diversity and altered composition in premenopausal women in the United States. Cancer Epidemiol Biomark Prev. 2015;24:546-54.

28. Kuczynski J, Stombaugh J, Walters WA, González A, Caporaso JG, Knight R. Using QIIME to analyze 16S rRNA gene sequences from microbial communities. Curr Protoc Bioinforma. 2011; Chapter: Unit 10.7. 
29. Edgar RC. Search and clustering orders of magnitude faster than BLAST. Bioinformatics. 2010;26:2460-1.

30. Navas-Molina JA, Peralta-Sánchez JM, González A, McMurdie PJ, Vázquez-Baeza Y, Xu Z, et al. Advancing our understanding of the human microbiome using QIIME. Methods Enzymol. 2013;531:371-444.

31. Quast C, Pruesse E, Yilmaz P, Gerken J, Schweer T, Yarza P, et al. The SILVA ribosomal RNA gene database project: improved data processing and web-based tools. Nucleic Acids Res. 2013;41:D590-6.

32. Schloss PD, Westcott SL, Ryabin T, Hall JR, Hartmann M, Hollister EB, et al. Introducing mothur: open-source, platformindependent, community-supported software for describing and comparing microbial communities. Appl Environ Microbiol. 2009;75:7537-41.

33. Langille MGI, Zaneveld J, Caporaso JG, McDonald D, Knights D, Reyes JA, et al. Predictive functional profiling of microbial communities using $16 \mathrm{~S}$ rRNA marker gene sequences. Nat Biotechnol. 2013;31:814-21.

34. Lozupone CKR. UniFrac: a new phylogenetic method for comparing microbial communities. Appl Env Microbiol. 2005;71:8228-35.

35. Mielke PW. 34 Meteorological applications of permutation techniques based on distance functions. Handb Stat. 1984;4:813-30.

36. Dufrene M, Legendre P. Species assemblages and indicator species: the need for a flexible asymmetrical approach. Ecol Monogr. 1997;67:345.

37. Benjamini Y, Hochberg Y. Controlling the false discovery rate: a practical and powerful approach to multiple testing. J R Stat Soc Ser B. 1995;57:289-300.

38. Fischbach FT, Dunning MB. A manual of laboratory and diagnostic tests. 8th ed. Philadelphia: Wolters Kluwer Health/Lippincott Williams \& Wilkins; 2009.

39. Ko Y-J, Kwon Y-M, Kim KH, Choi H-C, Chun SH, Yoon H-J, et al. High-sensitivity C-reactive protein levels and cancer mortality. Cancer Epidemiol Biomark Prev. 2012;21:2076-86.

40. Caporaso JG, Kuczynski J, Stombaugh J, Bittinger K, Bushman FD, Costello EK, et al. QIIME allows analysis of high-throughput community sequencing data. Nat Methods. 2010;7:335-6.

41. Oksanen J, Blanchet FG, Friendly M, Kindt R, Legendre P, McGlinn $\mathrm{D}$, et al. vegan: community ecology package. $\mathrm{R}$ package version 2.4-0. 2016.

42. Wickham H ggplot2: elegant graphics for data analysis. SpringerVerlag: New York; 2009.

43. Roberts DW. Ordination and multivariate analysis for ecology. R package version 1.5-0. 2012. Available at http://cran.r-project. org/package $=$ labdsv.

44. Turnbaugh PJ, Ridaura VK, Faith JJ, Rey FE, Knight R, Gordon JI. The effect of diet on the human gut microbiome: a metagenomic analysis in humanized gnotobiotic mice. Sci Transl Med. 2009;1:6ra14.

45. Cani PD, Bibiloni R, Knauf C, Waget A, Neyrinck AM, Delzenne $\mathrm{NM}$, et al. Changes in gut microbiota control metabolic endotoxemia-induced inflammation in high-fat diet-induced obesity and diabetes in mice. Diabetes. 2008;57:1470-81.

46. Cani PD, Possemiers S, Van de Wiele T, Guiot Y, Everard A, Rottier $\mathrm{O}$, et al. Changes in gut microbiota control inflammation in obese mice through a mechanism involving GLP-2-driven improvement of gut permeability. Gut. 2009;58:1091-103.

47. Ghoshal S, Witta J, Zhong J, de Villiers W, Eckhardt E. Chylomicrons promote intestinal absorption of lipopolysaccharides. J Lipid Res. 2009;50:90-7.

48. Amar J, Burcelin R, Ruidavets JB, Cani PD, Fauvel J, Alessi MC, et al. Energy intake is associated with endotoxemia in apparently healthy men. Am J Clin Nutr. 2008;87:1219-23.
49. Turnbaugh PJ, Ley RE, Mahowald MA, Magrini V, Mardis ER, Gordon JI. An obesity-associated gut microbiome with increased capacity for energy harvest. Nature. 2006;444:1027-31.

50. Turnbaugh PJ, Bäckhed F, Fulton L, Gordon JI. Diet-induced obesity is linked to marked but reversible alterations in the mouse distal gut microbiome. Cell Host Microbe. 2008;3:213-23.

51. Xu H, Barnes GT, Yang Q, Tan G, Yang D, Chou CJ, et al. Chronic inflammation in fat plays a crucial role in the development of obesity-related insulin resistance. J Clin Invest Am Soc Clin Investig. 2003;112:1821-30.

52. Cani PD, Possemiers S, Van de Wiele T, Guiot Y, Everard A, Rottier O, et al. Changes in gut microbiota control inflammation in obese mice through a mechanism involving GLP-2-driven improvement of gut permeability. Gut. 2009;58:1091-103.

53. Wong RG, Wu JR, Gloor GB. Expanding the UniFrac toolbox. PLoS ONE. 2016;11:e0161196.

54. Stenman LK, Burcelin R, Lahtinen S. Establishing a causal link between gut microbes, body weight gain and glucose metabolism in humans - towards treatment with probiotics. Benef Microbes. 2015;7:1-12.

55. Goodrich JK, Waters JL, Poole AC, Sutter JL, Koren O, Blekhman $\mathrm{R}$, et al. Human genetics shape the gut microbiome. Cell. 2014;159:789-99.

56. Lin H, An Y, Hao F, Wang Y, Tang H. Correlations of fecal metabonomic and microbiomic changes induced by high-fat diet in the pre-obesity state. Sci Rep. 2016;6:21618.

57. Beaumont M, Goodrich JK, Jackson MA, Yet I, Davenport ER, Vieira-Silva S, et al. Heritable components of the human fecal microbiome are associated with visceral fat. Genome Biol. 2016;17:189.

58. Wexler HM. Bacteroides: the good, the bad, and the nitty-gritty. Clin Microbiol Rev. 2007;20:593-621.

59. Walters WA, Xu Z, Knight R. Meta-analyses of human gut microbes associated with obesity and IBD. FEBS Lett. 2014;588:4223-33.

60. Schröder NWJ, Morath S, Alexander C, Hamann L, Hartung T, Zähringer U, et al. Lipoteichoic acid (LTA) of Streptococcus pneumoniae and Staphylococcus aureus activates immune cells via Toll-like receptor (TLR)-2, lipopolysaccharide-binding protein (LBP), and CD14, whereas TLR-4 and MD-2 are not involved. J Biol Chem. 2003;278:15587-94.

61. Mayerhofer R, Fröhlich EE, Reichmann F, Farzi A, Kogelnik N, Fröhlich E, et al. Diverse action of lipoteichoic acid and lipopolysaccharide on neuroinflammation, blood-brain barrier disruption, and anxiety in mice. Brain Behav Immun. 2017;60:174-87.

62. Kim KE, Cho YS, Baek KS, Li L, Baek K-H, Kim JH, et al. Lipopolysaccharide-binding protein plasma levels as a biomarker of obesity-related insulin resistance in adolescents. Korean Pediatr. 2016;59:231-8.

63. Watanabe Y, Nagai F, Morotomi M. Characterization of Phascolarctobacterium succinatutens sp. nov., an asaccharolytic, succinate-utilizing bacterium isolated from human feces. Appl Environ Microbiol. 2012;78:511-8.

64. Hosseini E, Grootaert C, Verstraete W, Van, de Wiele T. Propionate as a health-promoting microbial metabolite in the human gut. Nutr Rev. 2011;69:245-58.

65. Vinolo MAR, Rodrigues HG, Nachbar RT, Curi R. Regulation of inflammation by short chain fatty acids. Nutrients. 2011;3:858-76.

66. Arora T, Sharma R, Frost G. Propionate. Anti-obes satiety enhancing factor?. Appetite. 2011;56:511-5.

67. Navarro SL, Peterson S, Chen C, Makar KW, Schwarz Y, King IB, et al. Cruciferous vegetable feeding alters UGT1A1 activity: diet- and genotype-dependent changes in serum bilirubin in a controlled feeding trial. Cancer Prev Res. 2009;2:345-52. 
68. Morgan XC, Tickle TL, Sokol H, Gevers D, Devaney KL, Ward $\mathrm{DV}$, et al. Dysfunction of the intestinal microbiome in inflammatory bowel disease and treatment. Genome Biol. 2012;13:R79.

69. Machiels K, Joossens M, Sabino J, De Preter V, Arijs I, Eeckhaut $\mathrm{V}$, et al. A decrease of the butyrate-producing species Roseburia hominis and Faecalibacterium prausnitzii defines dysbiosis in patients with ulcerative colitis. Gut. 2014;63:1275-83.

70. van den Bogert B, de Vos WM, Zoetendal EG, Kleerebezem M. Microarray analysis and barcoded pyrosequencing provide consistent microbial profiles depending on the source of human intestinal samples. Appl Environ Microbiol. 2011;77:2071-80.

71. David LA, Maurice CF, Carmody RN, Gootenberg DB, Button JE, Wolfe BE, et al. Diet rapidly and reproducibly alters the human gut microbiome. Nature. 2014;505:559-63.

72. Wu GD, Chen J, Hoffmann C, Bittinger K, Chen Y-Y, Keilbaugh $\mathrm{SA}$, et al. Linking long-term dietary patterns with gut microbial enterotypes. Science. 2011;334:105-8. 